\title{
Research on a Pulse Interference Filter Used for the Fiber Bragg Grating Interrogation System
}

\author{
Weifang ZHANG ${ }^{1}$, Feifei REN ${ }^{1}$, Yingwu $\mathrm{LI}^{1}$, Bo JIN ${ }^{2}$, and Wei DAI ${ }^{1 *}$ \\ ${ }^{1}$ School of Reliability and Systems Engineering, Beihang University, Beijing 100191, China \\ ${ }^{2}$ School of Energy and Power Engineering, Beihang University, Beijing 100191, China \\ *Corresponding author: Wei DAI_E-mail: dw@buaa.edu.cn
}

\begin{abstract}
In this paper, a novel pulse interference filter for fiber Bragg grating (FBG) interrogation based on the tunable Fabry-Perot (F-P) filtering principle is proposed and experimentally demonstrated. The self-developed FBG interrogation system is devised for the aircraft health management of key structures. Nevertheless, the pulse interference is detected in the reflection spectrum of FBG causing interrogation system unstable. To address the problem, the first-order lag pulse broadening filter is proposed in this paper. The first-order lag filter is applied to preprocess and smooth the original signal, meanwhile enhancing the signal-to-noise ratio (SNR). Afterwards, peaks of reflection spectrum are distinguished with pulse interference by pulse broadening. Experimental results indicate that 634 peaks are detected before adopting the first-order lag pulse broadening filter. Comparatively, the number of peaks decreases to 203 after filtering the interference pulse, and the correct rate of peak detection is higher than $98.5 \%$. Through the comparison with the finite impulse response (FIR) filter, the advantage of first-order lag filter is proved. The vibration monitoring experiment demonstrates that this system has high dynamic precision with a dynamic interrogation range of $0 \mathrm{~Hz}-400 \mathrm{~Hz}$, and the maximum repetition rate of $800 \mathrm{~Hz}$.
\end{abstract}

Keywords: Fiber Bragg grating; tunable F-P filtering principle; pulse interference; digital filtering

Citation: Weifang ZHANG, Feifei REN, Yingwu LI, Bo JIN, and Wei DAI, "Research on a Pulse Interference Filter Used for the Fiber Bragg Grating Interrogation System,” Photonic Sensors, 2018, 8(3): 270-277.

\section{Introduction}

Fiber Bragg gratings (FBGs), by virtue of their inherent advantages, such as high sensitivity, compact size, low cost, and immunity to electromagnetic interference, have been applied widely in engineering fields [1-3]. They are utilized to measure the temperature or stress of structures for central wavelengths which can change synchronously $[4,5]$. Accordingly, the interrogation of central wavelengths is the foremost part in the application of FBGs [6]. However, a self-developed FBG interrogation system based on the tunable Fabry-Perot (F-P) filtering principle demonstrates that the pulse interference has great influence on the precision of interrogation. Therefore, a kind of digital filter suitable for FBG interrogation is demanded to solve the problem.

As for the digital filter, the moving average filter and conventional weighted average filter were

Received: 17 February 2018 / Revised: 17 May 2018

(C) The Author(s) 2018. This article is published with open access at Springerlink.com Springerlink.com

DOI: $10.1007 / \mathrm{s} 13320-018-0492-y$

Article type: Regular 
commonly employed to filter high-frequency interference in the previous research. However, they are not adept in inhibiting pulse interference [7-9]. A new weighted average filter that can eliminate pulse interference is proposed for the low-frequency signal. This method ranks the data at first, in succession removing the maximum and the minimum. The average of the rest data is utilized as the current value ultimately $[10,11]$. Therefore, this method is unsuitable for the real-time and high-frequency system on account of wasting a lot of system clock and resources. Raposo-Sanchez et al. [12] put forward a flat digital filter using alpha-splines. Several simulations have been done to prove it. Perraudin et al. [13] utilized a new model based on Wiener-type estimation to achieve the stationary signal processing on graphs. Chen et al. [14] applied a wavelet adaptive threshold algorithm to process noisy spectral signal of the FBG sensor system. These methods are too complex to be applied in FBG interrogation.

Based on all methods mentioned above that cannot be applied in FBG interrogation, the first-order lag pulse broadening filter is put forward in this paper to remove the pulse interference. It aims to enhance the accuracy of the interrogation system and strengthen the application of FBG in engineering.

\section{Methods}

\subsection{Tunable F-P filtering principle}

In this paper, the tunable F-P filtering principle is employed for the FBG interrogation $[15,16]$. It is one of the most important and widely utilized methods due to advantages of simplicity and high precision. Figure 1 presents the schematic diagram of the FBG interrogation system.

The F-P filter converts the optical signals of broadband light source into single wavelength signals. Spectrum of broadband light source and transmission spectrum of F-P filter are shown in Fig. 2. Afterwards, the single wavelength signals are divided into two parts by optical splitter to supply

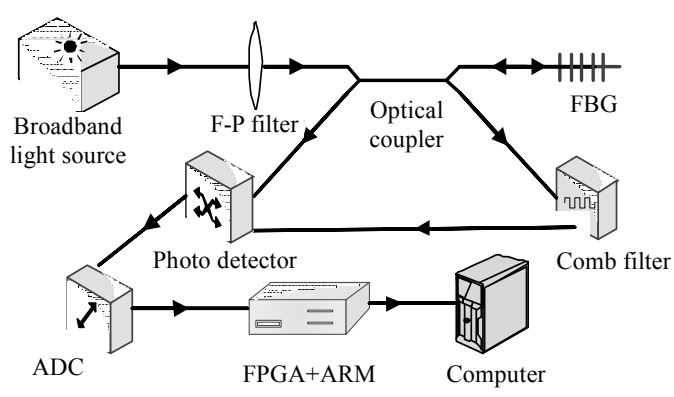

Fig. 1 Schematic diagram of FBG interrogation system.

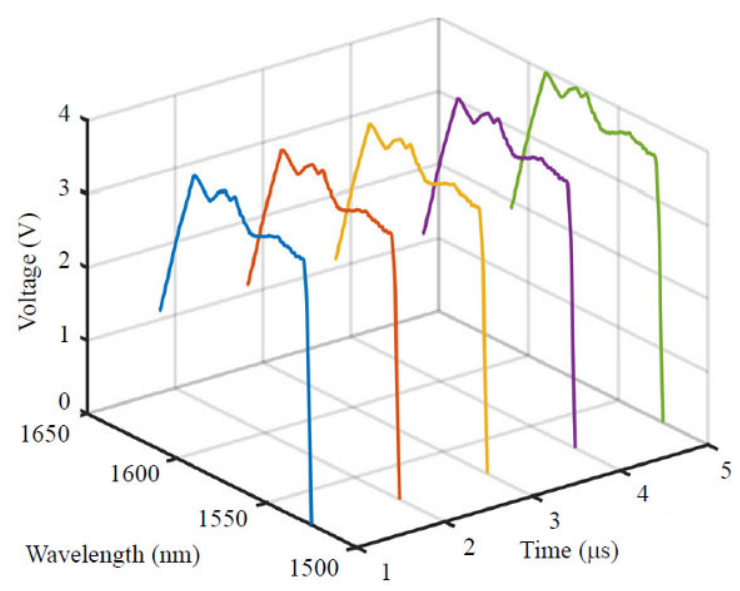

(a)

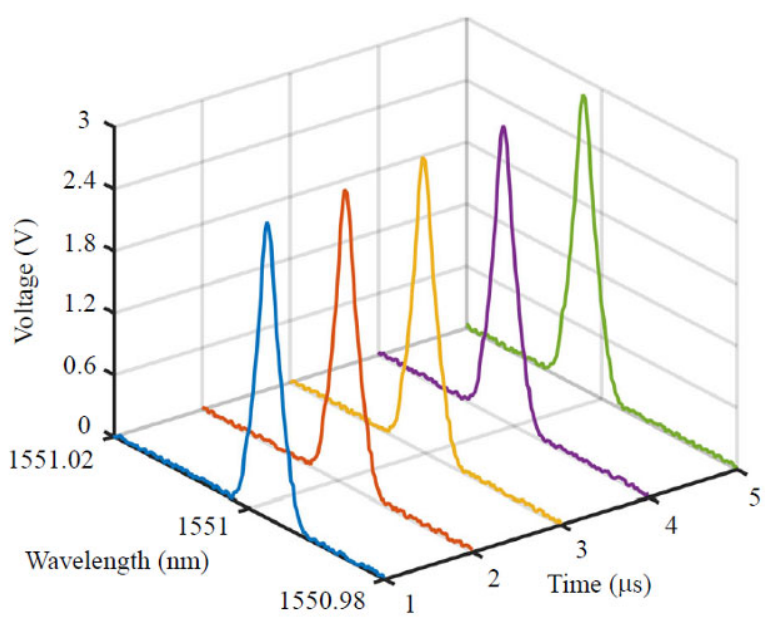

(b)

Fig. 2 Spectrum of broadband light source and F-P filter: (a) the spectrum of broadband light source and (b) the transmission spectrum of F-P filter.

the comb filter and FBG. The photodetector converts transmission spectrum of the comb filter and the reflection spectrum of the FBG into electrical signal. In succession, the electrical signal (analog signal) is sampled by analog-to-digital converter (ADC) to obtain the digital signal. For achieving better 
performance, the FPGA+ARM integrated platform is employed in this system. A field programmable gate array (FPGA) is applied to realize high speed sampling for high speed sampling, and an advanced reduced instruction set computer (RISC) machine (ARM) is employed to deal with the large amount of logical control and calculation for powerful computational capabilities $[17,18]$. Thus, the central wavelengths are capable of being calculated with the digital signal according to (1). The principle of the interrogation method is shown in Fig. 3.

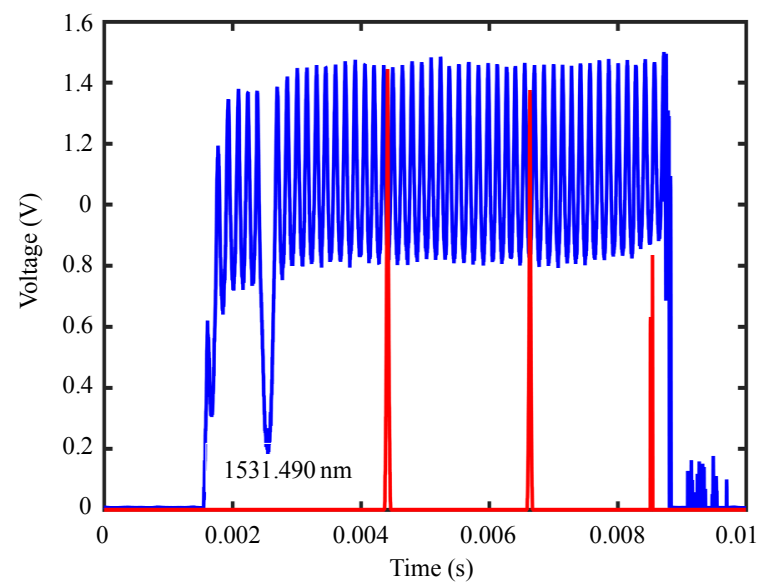

(a)

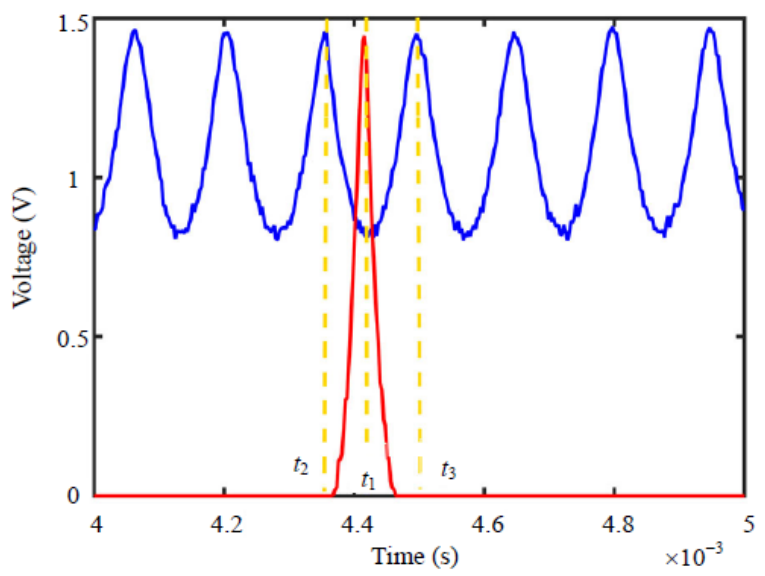

(b)

Fig. 3 Principle of interrogation method: (a) the original spectra of the FBG sensor and comb filter and (b) the detail diagram of the first grating in (a).

$$
\lambda_{\mathrm{FBG}}=\lambda_{k}+\left(\lambda_{k+1}-\lambda_{k}\right) \frac{t_{2}-t_{1}}{t_{2}-t_{3}}
$$

where $\lambda_{\mathrm{FBG}}$ is the central wavelength of the FBG, $t_{1}$ is the time of peak in comb filter transmission spectrum, $t_{2}$ denotes the time of peak in comb filter transmission spectrum next to $t_{1}, t_{3}$ expresses the position of peak in the FBG reflection spectrum $\left(t_{1} \leq t_{3} \leq t_{2}\right)$, and $\lambda_{k}$ and $\lambda_{k+1}$ represent the wavelengths corresponding to $t_{1}$ and $t_{2}$.

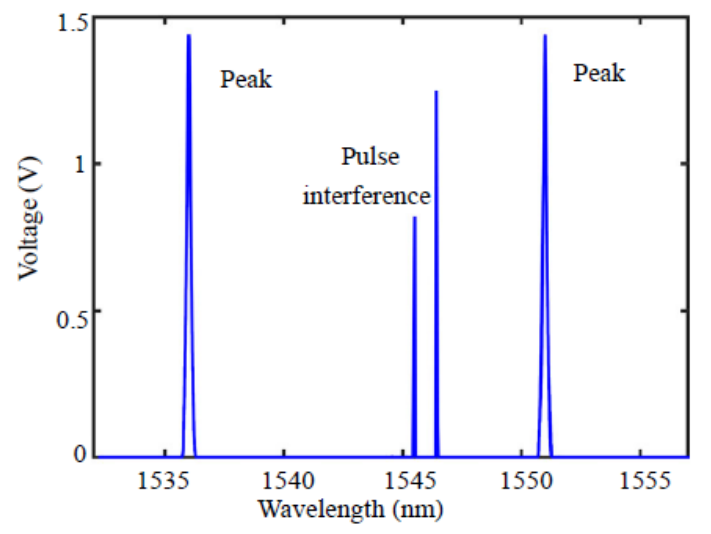

(a)

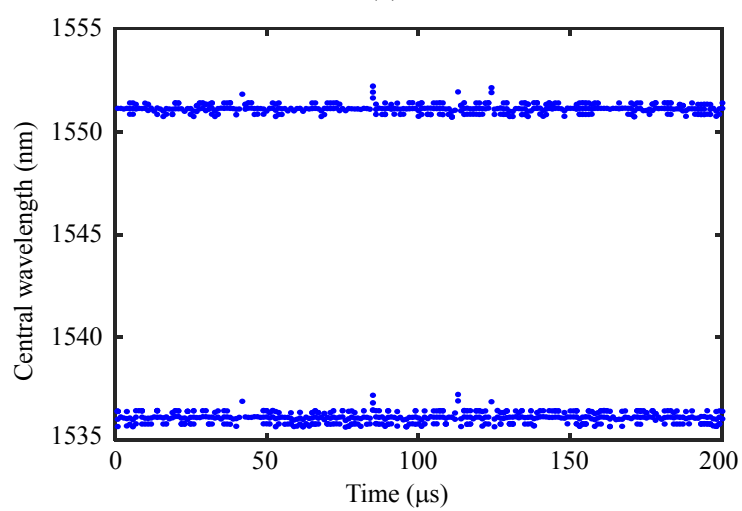

(b)

Fig. 4 Reflection spectrum of FBG and the results of interrogation: (a) the reflection spectrum of FBG and (b) the results of interrogation.

Based on the methods above, a self-developed interrogation system is established. It is experimentally indicated that the reflected spectrum of FBG has pulse signal. The pulse signal not only causes the existence of false central wavelengths but also has bad influence on the FBG interrogation precision. The reflection spectra of the FBG and interrogation results are shown in Fig. 4. The measurement results of 200 periods show that only 15 periods which includes 2 central wavelengths are correct. The correct rate of peak detection is $7.5 \%$. Consequently, the first-order lag pulse broadening filter is proposed to remove the pulse interference, thereby, improving the accuracy of central wavelengths. 


\subsection{First-order lag pulse broadening filter}

\subsubsection{First-order lag filter}

The first-order lag filter is employed to smooth the original signal accomplished by linear combination of current and previous values, as (2) presents. When $\alpha$ takes different values, $y_{n}$ varies with the change in $\alpha$, and the effect of $y_{n}$ is indicated in Fig. 5.

$$
y_{n}=(1-\alpha) X_{n}+\alpha y_{n-1}
$$

where $\alpha$ is the coefficient of the first-order lag filter, $y_{n}$ represents the result of filter, $X_{n}$ denotes current value, and $y_{n-1}$ expresses the previous value.

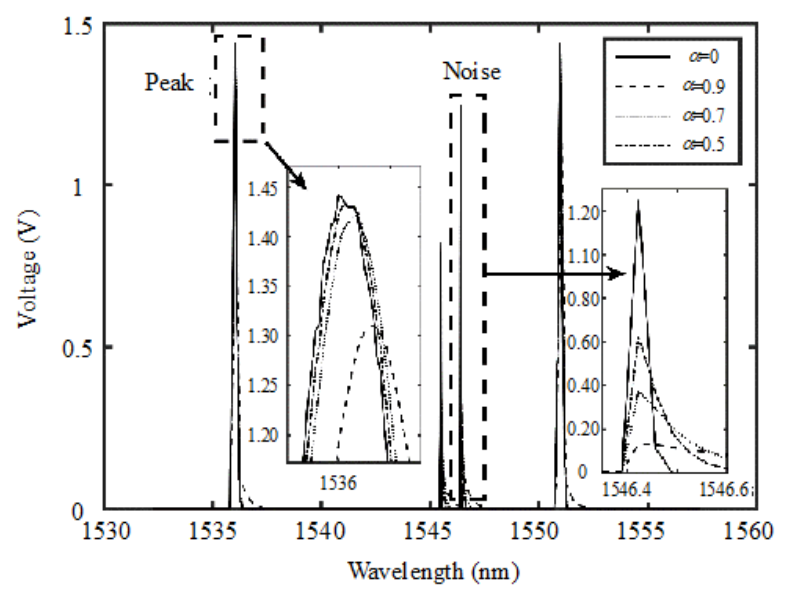

Fig. 5 Diverse results when $\alpha$ is different.

From the diagrams, the peak values of pulse signals decrease sharply with an increase in $\alpha$, simultaneously, hysteresis effect is more obvious. Taking hysteresis effect and SNR into consideration, the influence of the first-order lag filter can be written as (3). $\rho$ is comprised of SNR and hysteresis effect. It is proportional to SNR and inverse to the hysteresis effect. The larger $\rho$ is, the better the effect of the first-order lag filter is:

$$
\rho=\frac{20 \lg f_{\text {peak }} / f_{\text {pluse }}}{\delta}
$$

where $\rho$ is the characterization of effect, $f_{\text {peak }}$ is the peak value of reflection spectrum, $f_{\text {pluse }}$ represents the peak value of pulse interference, and $\delta$ expresses penalty factor caused by the shift of peak.

\subsubsection{Pulse broadening filter}

Except for the pulse interference, there are small signal noises in FBG reflection spectrum. Accordingly, the threshold should be set to judge the authenticity of peaks. The peaks of the reflection spectrum customarily tend to exist in the range within pulse broadening. Only when the maximum of width is greater than threshold, the authenticity of peak can be demonstrated. Thereupon, the maximum value and its position in this kind of range are recorded as the peaks of the reflection spectrum. In succession, the threshold of pulse broadening is put forward to distinguish the pulse interference with peaks of reflection spectrum. The definition of threshold for the pulse broadening can be described as (4). The peaks whose pulse broadening less than the threshold can be wiped off in real time.

$$
\Gamma=\frac{\gamma_{\max }+\gamma_{\min }}{2}
$$

where $\Gamma$ expresses the threshold of pulse broadening, $\gamma_{\max }$ is the maximum of pulse broadening, and $\gamma_{\min }$ is the minimum of pulse broadening.

\section{Experimental platform}

In the system, the FBG sensor with two central wavelengths is utilized, of which the model number is EDF-MP980 (type I). The properties of the FBG sensors are displayed in Table 1. The amplified spontaneous emission (ASE) broadband light source with the light signal among $1527.0 \mathrm{~nm}-1602.6 \mathrm{~nm}$ is adopted. The model number of F-P filter is TF2D4Z, and the scanning frequency is $800 \mathrm{~Hz}$. The versions of FPGA and ARM are XC7Z020-1CLG484I and CotrexA9, respectively. $\mathrm{ADC}$ is AD9244, and the speed is $5 \mathrm{MHz}$. The structure diagram of the interrogation system is shown in Fig. 6. The first-order lag pulse broadening filter is actualized in FPGA, which utilizes SPI protocol to control the data sending module driving F-P filter and ADC acquiring FBG signals. The data are transmitted from FPGA to ARM by AXI4 protocol, and then ARM accomplishes the 
interrogation calculation. Therefore, the central wavelength can be calculated in ARM. Meanwhile, the system using the above approaches is established as shown in Fig. 7.

Table 1 Properties of FBG sensors.

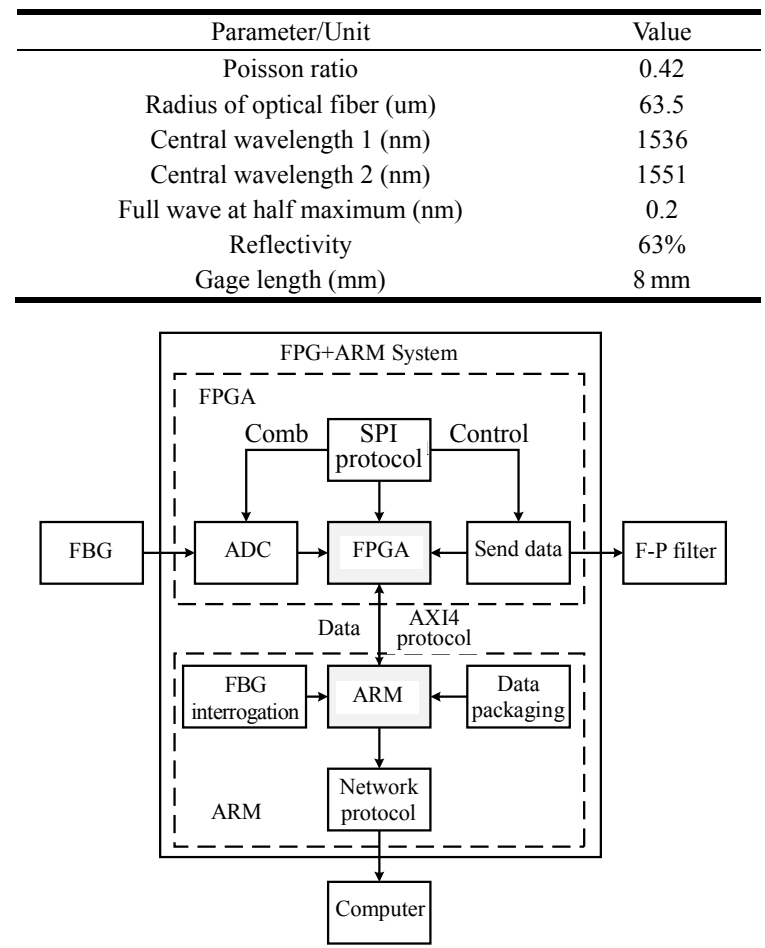

Fig. 6 Structure diagram of interrogation system.

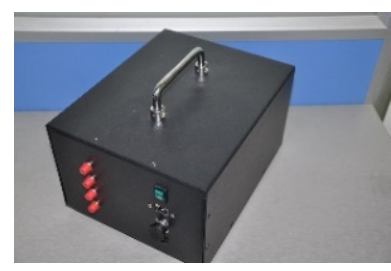

(a)

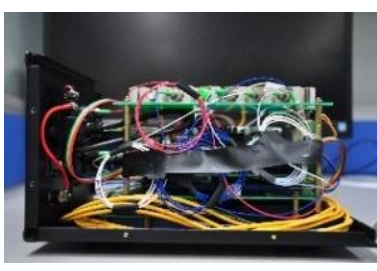

(b)
Fig. 7 FBG interrogation system: (a) external structure of the system and (b) internal structure of the system.

\section{Results and discussion}

The first-order lag filter is achieved by FPGA. Coefficient $\alpha$ can be obtained from Fig. 8(a). $\rho$ increases at first and falls in succession as the SNR and hysteresis effect increase. The best value of $\alpha$ is 0.4, which provides smaller hysteresis and acceptable SNR. When $\alpha$ is 0.4 , the effect of the first-order lag filter is depicted in Fig. 8(b), while the pulse interference still exists. Therefore, further filtering should be carried out.

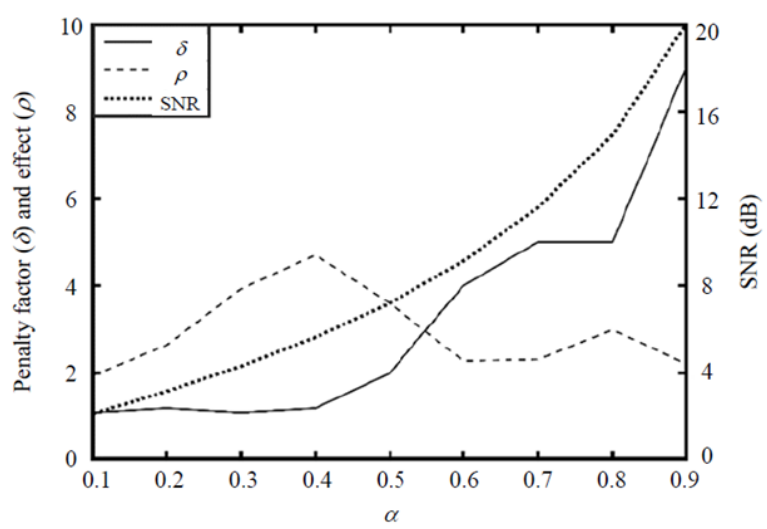

(a)

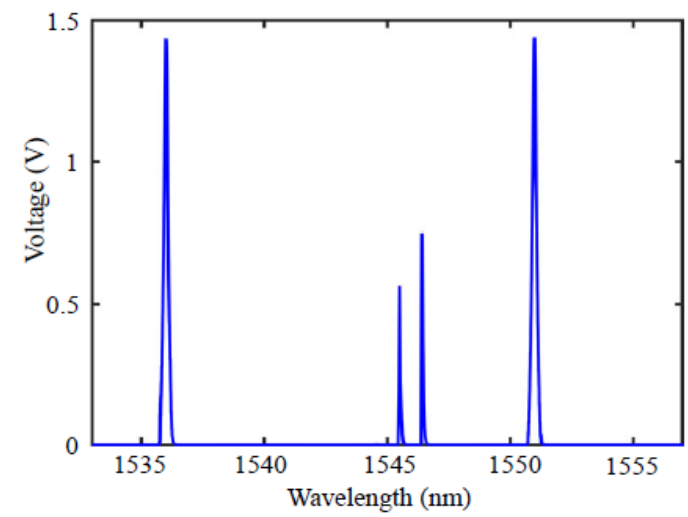

(b)

Fig. 8 Choose of $\alpha$ and the effect of the first-order lag filter: (a) $\rho$, SNR, and hysteresis effects change with $\alpha$ and (b) the effect of the first-order lag filter when $\alpha$ is fixed at 0.4.

The minimum of pulse broadening threshold is about 1 , and the maximum is around 800 . The pulse broadening of pulse interference is less than 200 , as well as greater than 500 in the true peaks of the reflection spectrum. Results of the pulse broadening calculated are presented in Fig. 9(a). Subsequently, the threshold of pulse broadening is fixed at 400 by using (4). The peaks with pulse broadening less than 400 can be regarded as pulse interference and removed. At room temperature $\left(26^{\circ} \mathrm{C}\right)$, the self-developed system is utilized to observe the effect of filtering pulse interference. Attributed to the first-order lag pulse broadening filter applied, the pulse interference in the reflection spectrum of FBG can be filtered. 203 peaks are detected in this experiment, and there are nearly 400 pulse signals. The correct rate of peak detection is higher than $98.5 \%$ compared with the actual number of peaks. 
Results of interrogation are shown in Fig.9(b). The accuracy of interrogation is improved significantly compared with the results before.

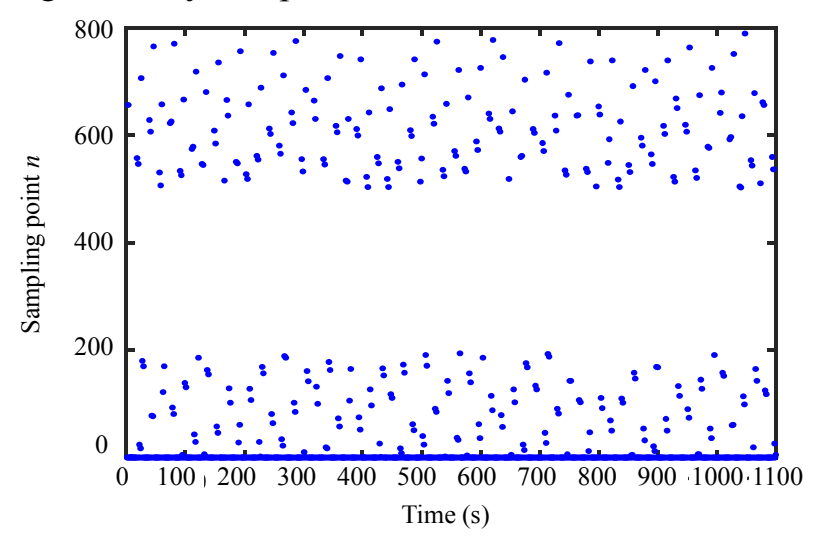

(a)

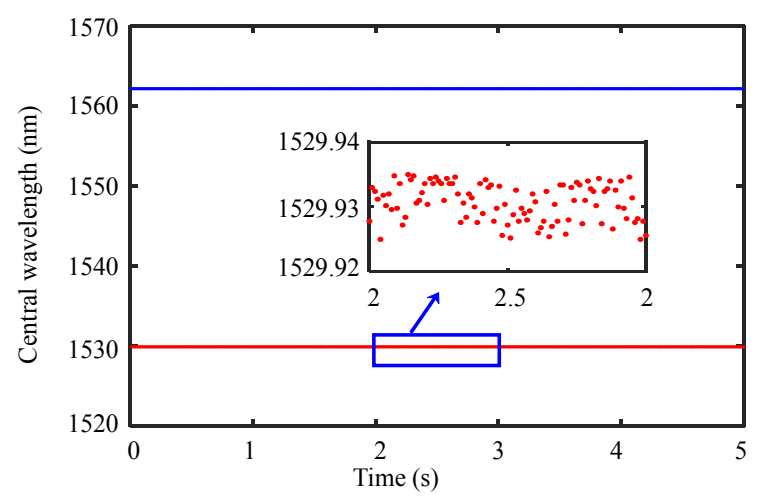

(b)

Fig. 9 Choice of pulse broadening threshold and the effect of the first-order lag pulse broadening filter: (a) the distribution of pulse broadening and (b) results of FBG interrogation after using the first-order lag pulse broadening filter.

For testing the filtering performance of the first-order lag pulse broadening filter, the FIR filter is utilized as contrast. The time domain difference equation of the finite impulse response (FIR) filter is shown in (5). This paper adopts Kaiser window with $\beta=7.865$ to cut the unit shock response function into finite sequence. The high frequency noise is higher than $50 \mathrm{kHz}$. Thus, the FIR filter is designed with a $50 \mathrm{kHz}$ cut-off frequency. After simulation, $h(\mathrm{~m})$ is calculated as Fig. 10(a), and the size of it is 150 $(N=150)$. The amplitude-frequency curve of FIR filter is shown in Fig. 10(b), and the designed FIR filter has an attenuation stopband of $80 \mathrm{~dB}$.

$$
\gamma(n)=\sum_{m=0}^{N-1} h(m) \times(n-m)
$$

where $\chi(n)$ is the result of discrete-time filter, $n$ is the serial number of $\chi(n), h(m)$ is unit shock response function, $m$ is the serial number of $h(m), N$ is the size of $h(m)$, and $x(n-m)$ is the input signal sequence.

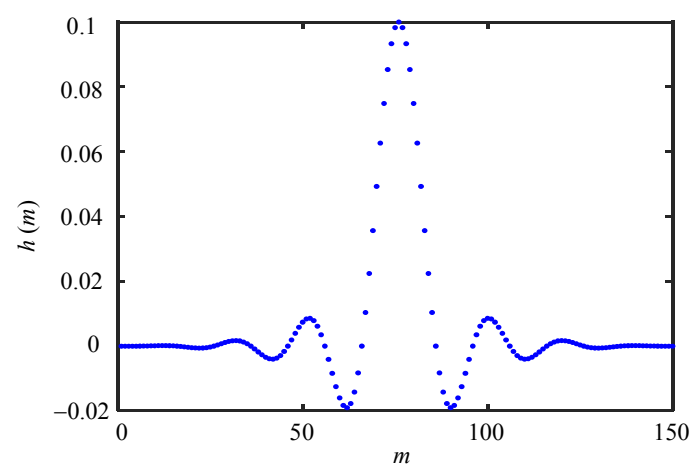

(a)

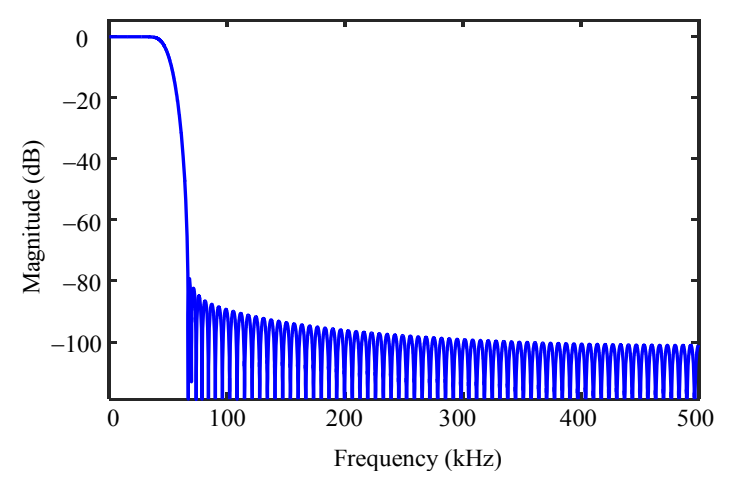

(b)

Fig. 10 FIR filter designed in this paper: (a) the $h(\mathrm{~m})$ calculated with $m$ and (b) the amplitude-frequency curve of FIR filter.

To compare the filtering performances of the first-order lag pulse broadening filter and FIR filter, a sample of data are applied in simulation with the $1000 \mathrm{kHz}$ sampling frequency. Original reflection spectrum and the signal processed by two kinds of filters are sketched in Fig. 11. The first-order lag filer and FIR filter can smooth the spectrum and inhibit the high frequency noise. The performances for inhibiting the pulse interference of two kinds of filter are basically the same. Amplitude of pulse interference in original spectrum is compressed to about one-tenth by two kinds of filters. The hysteresis effect caused by FIR filter is far greater than the first-order lag filer. Meanwhile, the FIR filter is more difficult for program implementation than the first-order lag filter as the obtaining of $h(m)$ 
needs a lot of calculations. At the same time, the filtering process occupies a huge storage space when $N$ is large. Therefore, the first-order lag pulse broadening filter tends to gain greater effect in this interrogation system. In short, the first-order lag pulse broadening filter not only performs well in inhibiting high frequency impulse noise but also has better computational performance.

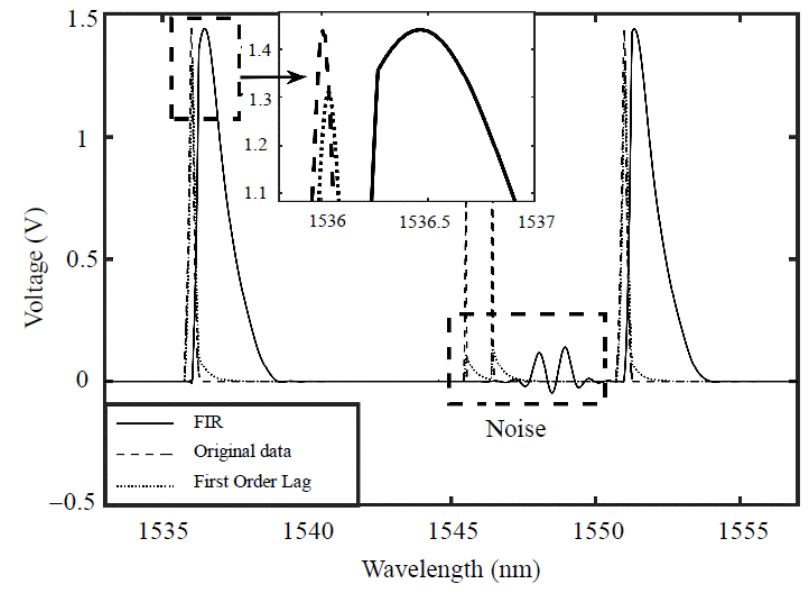

Fig. 11 Contrast of original signal and filtered signal.

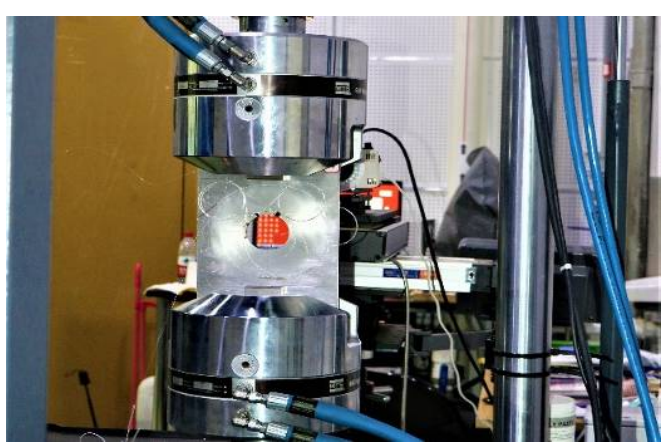

(a)

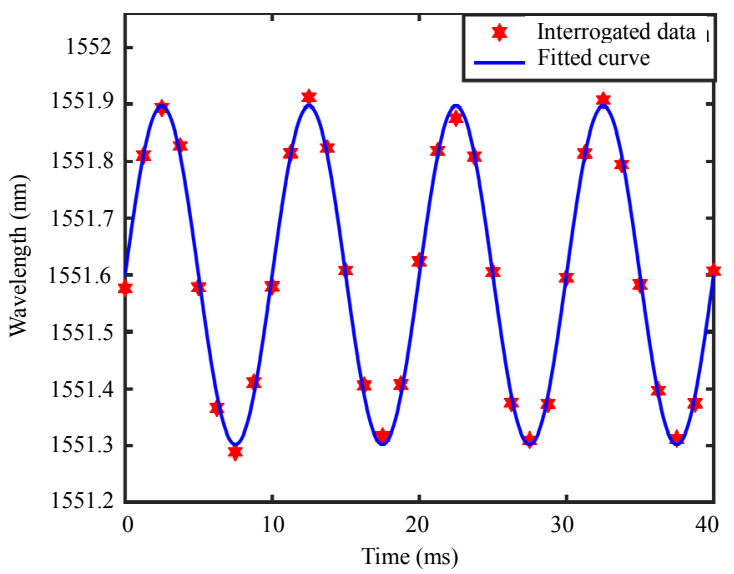

(b)

Fig. 12 Device and monitoring results of dynamic experiment: (a) the experimental device and (b) the experimental results.
For testing the performance of this system, an FBG sensor is pasted on an aluminum alloy plate and $100 \mathrm{~Hz}$ sinusoidal force is applied via the fatigue test machine. The ambient temperature of experiment is $26{ }^{\circ} \mathrm{C}$. The device is shown in Fig. 12(a), and the results are shown in Fig. 12 (b).

The central wavelength of the FBG sensor is calculated via interrogation system and transmitted to computer for further processing. In Fig. 12(b), the measured data and fitted curve are provided, which illustrate that the system has high dynamic precision. What's more, as the maximum scan frequency of F-P filter is $800 \mathrm{~Hz}$, the maximum repetition rate of this system is $800 \mathrm{~Hz}$ when one FBG is measured. Thus, the maximum frequency of signal that this system can measure is $400 \mathrm{~Hz}$ according to the sampling theorem.

\section{Conclusions}

The existence of pulse interference in the FBG interrogation system causes the low accuracy of central wavelengths and limits the applications of FBG. In this study, a novel and practical pulse interference filter that is the first-order lag pulse broadening filter for FBG interrogation based on the tunable F-P filtering principle is designed. 603 peaks are detected as well as the stability and the accuracy of interrogation is influenced seriously. To inhibit the pulse interference, the first-order lag pulse broadening filter is utilized. $\alpha$ is 0.4 , and $\Gamma$ is 400. Experimental results show that about 400 pseudo peaks are detected. Furthermore, another comparison experiment with FIR filter proves that the first-order lag pulse broadening filter has better computational performance. Meanwhile, a vibration monitoring experiment is carried to test the performance of this system. Results demonstrate that this system has high dynamic precision and a maximum repetition rate of $800 \mathrm{~Hz}$. The dynamic interrogation range is $0 \mathrm{~Hz}-400 \mathrm{~Hz}$. In conclusion, the method proposed in this paper is useful for removing the pulse interference, and it improves the performance in FBG interrogation, which has a wide range of applications. 


\section{Acknowledgment}

This work was supported by the Technical Foundation Program (Grant Nos. JSZL 2014601 B001 and JSZL 2017601 C002) from the Ministry of Industry and Information Technology of China.

Open Access This article is distributed under the terms of the Creative Commons Attribution 4.0 International License (http://creativecommons.org/licenses/by/4.0/), which permits unrestricted use, distribution, and reproduction in any medium, provided you give appropriate credit to the original author(s) and the source, provide a link to the Creative Commons license, and indicate if changes were made.

\section{References}

[1] V. Schukar, N. Kusche, G. Kalinka, and W. Habel, "Field deployable fiber Bragg grating strain patch for long-term stable health monitoring applications," Applied Sciences, 2013, 3(1): 39-54.

[2] S. F. Wang, T. H. Chen, L. Tsai, C. C. Huang, and C. C. Chiang, "A FBG intensity modulation system combined with an optical whispering gallery mode edge filter," Applied Sciences, 2016, 6(4): $1-10$.

[3] X. J. Lv, X. F. Zhao, L. Wang, H. Dong, and Y. F. Zhu, "Research on fiber Bragg grating sensing technique for cable tension monitoring of suspension bridges," Applied Mechanics \& Materials, 2013, 368-370: 1391-1395.

[4] Y. Liu, Y. M. Li, F. Zhou, and Y. Zhong, "Analysis on strain sensing characteristic of long period fibre grating based on deep-grooved process," Optica Acta International Journal of Optics, 2017, 64(7): 672-680.

[5] T. T. Yang, X. G. Qiao, Q. Z. Rong, and W. J. Bo, "Fiber Bragg gratings inscriptions in multimode fiber using $800 \mathrm{~nm}$ femtosecond laser for high-temperature strain measurement," Optics \& Laser Technology, 2017, 93: 138-142.

[6] T. Jiang, L. Ren, D. S. Li, X. Cheng, and Z. G. Jia, "Application of FBG strain hoop sensor in pipeline safety monitoring research," Journal of Optoelectronics • Laser, 2015, 26(8): 1536-1542.

[7] W. Y. Li, G. L. Wei, F. Han, and Y. R. Liu, "Weighted average consensus-based unscented Kalman filtering," IEEE Transactions on
Cybernetics, 2016, 46(2): 558-567.

[8] E. Isufi, A. Loukas, A. Simonetto, and G. Lenus, "Autoregressive moving average graph filtering," IEEE Transactions on Signal Processing, 2016, 65(2): 274-288.

[9] J. Nikonowicz and M. Jessa, "A novel method of blind signal detection using the distribution of the bin values of the power spectrum density and the moving average," Digital Signal Processing, 2017, 66: $18-28$.

[10] Q. Chen, W. C. Wang, C. Yin, X. X. Jin, and J. Zhou, "Distributed cubature information filtering based on weighted average consensus," Neurocomputing, 2017, 243: 115-124.

[11] Y. Wang, J. Y. Wang, X. Song, and L. Hang, “An efficient adaptive fuzzy switching weighted mean filter for salt-and-pepper noise removal," IEEE Signal Processing Letters, 2016, 23(11): 1582-1586.

[12] M. Á. Raposo-Sánchez, J. Sáez-Landete, and F. Cruz-Roldán, "Analog and digital filters with $\alpha$-splines," Digital Signal Processing, 2017, 66: 1-9.

[13] N. Perraudin and P. Vandergheynst, "Stationary signal processing on graphs," IEEE Transactions on Signal Processing, 2017, 65(13): 3462-3477.

[14] Y. Chen, Y. N. Cheng, and H. L. Liu, “Application of improved wavelet adaptive threshold de-noising algorithm in FBG interrogation," Optik, 2017, 132: 243-248.

[15] A. Pospori and D. J. Webb, "Stress sensitivity analysis of optical fiber Bragg grating based Fabry-Pérot interferometric sensors," Journal of Lightwave Technology, 2017, 35(13): 2654-2659.

[16] Q. Z. Rong, Z. H. Shao, X. L. Yin, T. T. Gang, F. Liu, A. Sun, et al., "Ultrasonic imaging of seismic physical models using fiber Bragg grating Fabry-Perot probe," IEEE Journal of Selected Topics in Quantum Electronics, 2017, 23(2): 1-6.

[17] L. Costas, R. Fernández-Molanes, J. J. Rodríguez-Andina, and J. Farina, "Characterization of FPGA-master ARM communication delays in zynq devices," in Proceeding of IEEE International Conference on Industrial Technology, Toronto, Canada, 2017, pp. 942-947.

[18] L. F. Xiao, X. G. Chen, and B. H. Lin, "Design and realization of strain measurement system based on FPGA and ARM," in Proceeding of IEEE International Conference on Intelligent Control \& Information Processing, Beijing, China, 2013, pp. 853-857. 